\title{
Configuration entropy for quarkonium in a finite density plasma
}

\author{
Nelson R. F. Braga $\odot^{*}$ and Rodrigo da Mata ${ }^{\dagger}$ \\ Instituto de Física, Universidade Federal do Rio de Janeiro, Caixa Postal 68528, RJ 21941-972, Brazil
}

(Received 12 March 2020; accepted 18 May 2020; published 26 May 2020)

\begin{abstract}
In recent years, many examples appeared in the literature where the configuration entropy (CE), introduced by Gleiser and Stamatopoulos, plays the role of an indicator of stability of physical systems. It was observed that, comparing states of the same system, the lower is the value of the CE, the more stable is the state. In this work, we investigate the behavior of the differential configuration entropy (DCE), that is appropriate for systems with continuous degrees of freedom, in a new context. We consider quasistates of quarkonium (a vector meson made of a heavy quark antiquark pair) inside a plasma at finite density. It is known that the density increases the dissociation effect for quasiparticles inside a plasma. So, increasing the density of a thermal medium corresponds to reducing the stability of the quasiparticles. In order to investigate how this situation is translated in the configuration entropy context, we use a recently developed holographic anti-de Sitter/QCD model for heavy vector mesons. The quasinormal modes describing the quasistates are obtained and the corresponding DCE is calculated. We find, for bottomonium and charmonium $1 S$ quasi-states, that the DCE increases with the quark density, or quark chemical potential, of the medium. This result shows that the DCE works again as an indicator of stability, represented in this case by the dissociation effect associated with the density.
\end{abstract}

DOI: 10.1103/PhysRevD.101.105016

\section{INTRODUCTION}

An interesting tool to investigate the stability of physical systems is the configuration entropy (CE), introduced by Gleiser and Stamatopoulos in Refs. [1,2] (see also [3]). An increase in the value of the CE is associated with a decrease in the stability. Such a behavior was observed in many different physical systems, such as compact astrophysical objects [4] and holographic anti-de Sitter/QCD models [5-10]. There are other many interesting applications of configuration entropy in the literature, as, for example, [11-30].

The purpose of the present paper is to investigate the application of the configuration entropy to a physical system of great interest currently: heavy mesons inside a quark gluon plasma with finite density. The interest in such a system comes from the quark gluon plasma (QGP). This very short-lived state of matter, where quarks and gluons are not confined, is produced in heavy ion collisions and consists of a strongly interacting thermal medium. It is a highly nontrivial task to build up a picture of the QGP from the particles that reach the detectors. For reviews about

\footnotetext{
*braga@if.ufrj.br

†rsilva@if.ufrj.br
}

Published by the American Physical Society under the terms of the Creative Commons Attribution 4.0 International license. Further distribution of this work must maintain attribution to the author(s) and the published article's title, journal citation, and DOI. Funded by SCOAP ${ }^{3}$.
QGP, see, for example, [31-34]. One of the important available sources of information is the abundance of heavy vector mesons, made of $c \bar{c}$ or $b \bar{b}$ quarks in the final products of heavy ion collisions. These particles are partially dissociated in the plasma and their degree of dissociation depends on the temperature and density of the plasma. So, it is possible to relate their relative abundance with the properties of the preexisting medium.

It is possible to describe the thermal behavior of heavy vector mesons inside a plasma using holographic models [35-39]. The dissociation of charmonium and bottomonium is represented in these references as the decrease in the peaks of the thermal spectral functions, that represent the amplitude of finding a particle with a given energy inside the medium. This dissociation process can alternatively be analyzed through the quasinormal modes, that are normalizable solutions, with complex frequencies, for the fields that describe the mesons. The real parts of the frequencies are related to the masses and the imaginary parts to the widths of the quasistates. In Refs. [40-42], quasinormal modes for heavy vector mesons were studied using the holographic model of $[38,39]$.

The configuration entropy [3] was motivated by the wellknown information entropy of Shannon [43] that is defined for a discrete variable $x$ that may have the values $x_{n}$ with probabilities $p_{n}$ as

$$
-\sum_{n} p_{n} \log p_{n}
$$


and measures the amount of information one gains in getting to know the value of $x$. Note that the probabilities satisfy the normalization condition $\sum_{n} p_{n}=1$.

The continuous version of Eq. (1), in position space, reads

$$
S=-\int d^{d} r \epsilon(\vec{r}) \log \epsilon(\vec{r})
$$

where

$$
\epsilon(\vec{r})=\frac{|\rho(\vec{r})|^{2}}{\int d^{d} r|\rho(\vec{r})|^{2}}
$$

is a normalized funtion $\int d^{d} r \epsilon(\vec{r})=1$, called (spatial) modal fraction. In order to introduce the configuration entropy, one considers the momentum space version, by Fourier transforming

$$
\tilde{\rho}(\vec{k})=\frac{1}{(2 \pi)^{d / 2}} \int d^{d} r \rho(\vec{r}) \exp (-i \vec{k} \cdot \vec{r})
$$

The CE is defined as

$$
\tilde{S}=-\int d^{d} k \tilde{\epsilon}(\vec{k}) \log \tilde{\epsilon}(\vec{k}),
$$

where

$$
\tilde{\epsilon}(\vec{k})=\frac{|\tilde{\rho}(\vec{k})|^{2}}{\int d^{d} k|\tilde{\rho}(\vec{k})|^{2}}
$$

is the (momentum space) modal fraction, that is also normalized: $\int d^{d} k \tilde{\epsilon}(\vec{k})=1$.

Information entropies like $S$ and $\tilde{S}$, based in conjugate variables in the sense of Eq. (4), satisfy the so-called entropic uncertainty relation $[44,45]$ that, for this d-dimensional case takes the form

$$
S+\tilde{S} \geq d(1+\log (\pi))
$$

So, one could guess that a variation of the configuration entropy, defined in momentum space, $\tilde{S}$ could be associated with a particular variation of the conjugate quantity, $S$, defined in position space. Such a conjecture was investigated recently in Ref. [45], where it was found that, for the case of an anti-de Sitter black hole, when the temperature varies, both $\tilde{S}$ and $S$ vary but their sum remains constant.

For continuum systems, like field theories, it was pointed out in Ref. [22] that a different kind of configuration entropy should be used. In order to avoid negative values, or singularities in the entropy, one should replace the (momentum) modal fraction of Eq. (6) by

$$
\widetilde{D \epsilon}(\vec{k})=\frac{|\tilde{\rho}(\vec{k})|^{2}}{|\tilde{\rho}(\vec{k})|_{\max }^{2}}
$$

where $|\tilde{\rho}(\vec{k})|_{\max }^{2}$ is the maximum value of $|\tilde{\rho}(\vec{k})|^{2}$. Then one introduces the differential configuration entropy (DCE) as

$$
\tilde{S}=-\int d^{d} k \widetilde{D \epsilon}(\vec{k}) \log \widetilde{D \epsilon}(\vec{k}) .
$$

This is the entropy that we will calculate in this paper. For the dual entropy, one can follow the same idea and define

$$
D \epsilon(\vec{r})=\frac{|\rho(\vec{r})|^{2}}{|\rho(\vec{r})|_{\max }^{2}}
$$

and a corresponding quantity

$$
S=-\int d^{d} r \operatorname{D\epsilon }(\vec{r}) \log D \epsilon(\vec{r}) .
$$

We will look at this dual quantity in order to see if a behavior similar to the black hole case [45] is found. However, it is important to remark that the DCE of Eq. (9) and the dual quantity of Eq. (11) are not subject to the relation (7) since $|\tilde{\rho}(\vec{k})|^{2}$ and $|\rho(\vec{r})|_{\max }^{2}$ are not constants.

This paper is organized in the following way: in Sec. II, we review the holographic model for heavy vector mesons in a plasma. In Sec. III, we develop the calculation of DCE for charmonium and bottomonium $1 \mathrm{~S}$ states. Then, in Sec. IV, we present and analyze the results obtained, and finally, in Sec. V, we present our summary and conclusions.

\section{HOLOGRAPHIC HEAVY VECTOR MESONS AT FINITE DENSITY}

Heavy vector mesons are described holographically $[38,39]$ by a vector field $V_{m}=\left(V_{\mu}, V_{z}\right)(\mu=0,1,2,3)$, living in a five-dimensional curved space, that is assumed to be dual to the four-dimensional gauge theory current $J_{\mu}=\bar{\psi} \gamma^{\mu} \psi$. The curved five-dimensional space is just an anti-de Sitter space for the case when the mesons are in the vacuum (vanishing temperature and density). Additionally, there is a scalar background. The action reads

$$
I=\int d^{4} x d z \sqrt{-g} e^{-\phi(z)}\left\{-\frac{1}{4 g_{5}^{2}} F_{m n} F^{m n}\right\},
$$

where $F_{m n}=\partial_{m} V_{n}-\partial_{n} V_{m}$. The background scalar field $\phi(z)$ has the form

$$
\phi(z)=\kappa^{2} z^{2}+M z+\tanh \left(\frac{1}{M z}-\frac{\kappa}{\sqrt{\sigma}}\right) .
$$

The parameter $\sigma$, with dimension of energy squared, represents effectively the string tension of the strong quark 
antiquark interaction. The mass of the heavy quarks is represented by $\kappa$. The third parameter, $M$, has a more subtle interpretation. Heavy vector mesons undergo nonhadronic decay processes, when the final state consists of light leptons, like an $e^{+} e^{-}$pair. In such transitions, there is a very large mass change of the order of the meson mass. The parameter $M$ represents effectively the mass scale of such a transition, characterized by a matrix element $\left\langle 0\left|J_{\mu}(0)\right| n\right\rangle=\epsilon_{\mu} f_{n} m_{n}$, where $f_{n}$ is the decay constant, $|n\rangle$ is a meson state at radial excitation level $n$ with mass $m_{n},|0\rangle$ is the hadronic vacuum, and $J_{\mu}$ the hadronic current. The values that provide the best fit to charmonium and bottomonium spectra of masses and decay constants at zero temperature are, respectively,

$$
\begin{aligned}
& \kappa_{c}=1.2, \quad \sqrt{\sigma_{c}}=0.55, \quad M_{c}=2.2 ; \\
& \kappa_{b}=2.45, \quad \sqrt{\sigma_{b}}=1.55, \quad M_{b}=6.2 \text {, }
\end{aligned}
$$

where all quantities are expressed in $\mathrm{GeV}$. The geometry dual to a finite temperature medium is in general a black hole one. For the case when the medium additionally has a finite chemical potential $\mu$, the black hole has charge [46-48]. In particular, it is a five-dimensional anti-de Sitter charged black hole space with metric

$$
d s^{2}=\frac{R^{2}}{z^{2}}\left(-f(z) d t^{2}+d \vec{x} \cdot d \vec{x}+\frac{d z^{2}}{f(z)}\right)
$$

where

$$
f(z)=1-\frac{z^{4}}{z_{h}^{4}}-q^{2} z_{h}^{2} z^{4}+q^{2} z^{6}
$$

and $f\left(z_{h}\right)=0$. The relation between the horizon position $z_{h}$ and the temperature $T$ of the black hole is obtained requiring that there is no conical singularity at the horizon,

$$
T=\frac{\left|f^{\prime}(z)\right|_{\left(z=z_{h}\right)}}{4 \pi}=\frac{1}{\pi z_{h}}-\frac{q^{2} z_{h}^{5}}{2 \pi} .
$$

The parameter $q$, proportional to the black hole charge, is related to the density of the medium, or quark chemical potential, $\mu$ of the gauge theory. The quantity $\mu$ works as the source of correlators of the quark density operator $\bar{\psi} \gamma^{0} \psi$. So, it should appear in the Lagrangian multiplying the quark density. In the holographic description, the time component $V_{0}$ of the vector field plays this role. So, one considers a particular solution for the vector field $V_{m}$ with only one nonvanishing component $V_{0}=A_{0}(z)\left(V_{z}=0\right.$, $V_{i}=0$ ). Assuming that the relation between $q$ and $\mu$ is the same as in the case of no background, that means $\phi(z)=0$, the solution for the time component of the vector field is $A_{0}(z)=c-q z^{2}$, where $c$ is a a constant. Imposing $A_{0}(0)=\mu$ and $A_{0}\left(z_{h}\right)=0$, one finds

$$
\mu=q z_{h}^{2}
$$

So, specifying both $z_{h}$ and $q$, the values of the temperature and the chemical potential are fixed and contained into the metric (15).

It is interesting to mention that there are many interesting previous studies using holography to describe thermal effects and heavy flavors like, for example, [49-64].

\section{CONFIGURATION ENTROPY OF THE HEAVY MESONS}

\section{A. Energy density}

The quantity that is relevant for the determination of the configuration entropy of heavy mesons is the energy density, that is the $T_{00}$ component of the energy momentum tensor. We assume that in this phenomenological model $T_{m n}$ is obtained from the action in the same way as in general relativity. That means writing the action as $\int d^{4} x d z \sqrt{-g} \mathcal{L}$ the energy momentum tensor has the form

$$
T_{m n}(z)=\frac{2}{\sqrt{-g}}\left[\frac{\partial(\sqrt{-g} \mathcal{L})}{\partial g^{m n}}-\frac{\partial}{\partial x^{p}} \frac{\partial(\sqrt{-g} \mathcal{L})}{\partial\left(\frac{\partial g^{m n}}{\partial x^{p}}\right)}\right] .
$$

So, for the action (12), the energy density for the vector field is

$\rho(z)=\frac{e^{-\phi(z)}}{g_{5}^{2}}\left[g_{00}\left(\frac{1}{4} g^{m p} g^{n q} F_{m n} F_{p q}\right)-g^{m n} F_{0 n} F_{0 m}\right]$.

Considering the metric (15) and a solution corresponding to a meson at rest in the $x^{\mu}$ directions $V_{\mu}=\eta_{\mu} v(p, z) e^{-i \omega t}$, with $\eta_{\mu}=(0,1,0,0)$, the energy density takes the form

$$
\rho(z)=\frac{z^{2} e^{-\phi(z)}}{2 R^{2} g_{5}^{2}}\left[|\omega|^{2}|v|^{2}+f^{2}\left|\partial_{z} v\right|^{2}\right] .
$$

In order to obtain the energy density for a meson inside the plasma, one has to find the solution for the field $v$ representing the corresponding quasistate and plug it into Eq. (21). At zero temperature, states are represented holographically by normalizable solutions of the gravity field equations. These types of solutions are called normal modes and satisfy trivial boundary conditions. On the other hand, at finite temperature, the solutions that represent the quasistates are the so-called quasinormal modes, that are also normalizable solutions of the field $v$ but satisfy nontrivial boundary conditions. At finite $T$, there is an event horizon at $z=z_{h}$ where one has to impose infalling boundary conditions. Additionally, the normalizability condition requires that the fields vanish at the boundary $z=0$. Satisfying both conditions requires, in general, solutions corresponding to complex frequencies $\omega$. The real part, $\operatorname{Re}(\omega)$, is related to the thermal mass and the 
imaginary part, $\operatorname{Im}(\omega)$, is related to the thermal width. We will see in the next section how to obtain these solutions.

\section{B. Quasinormal modes}

As in the previous section, we consider $V_{z}=0$ and $V_{\mu}=\eta_{\mu} v(p, z) e^{-i \omega t}$, with $\eta_{\mu}=(0,1,0,0)$. Introducing the electric field component $E=\omega V_{1}$, the equations of motion coming from action (12) with the metric (15) take the form

$$
E^{\prime \prime}+\left(\frac{f^{\prime}}{f}-\frac{1}{z}-\phi^{\prime}\right) E^{\prime}+\frac{\omega^{2}}{f^{2}} E=0,
$$

where (') represents derivative with respect to the radial $\mathrm{z}$ coordinate.

One has to impose the normalizability condition at $z=0$ and the infalling condition at $z=z_{h}$. It is convenient, in order to impose the boundary conditions at the horizon, to rewrite the field equations in such a way that they separate into a combination of infalling and outgoing waves. One introduces the coordinate $r_{*}$, implicitly defined by the relation $\partial_{r_{*}}=-f(z) \partial_{z}$ with $r_{*}(0)=0$ for $\mathrm{z}$ in the $0 \leq z \leq z h$. In addition, let us introduce the field

$$
\psi=e^{-\frac{B(z)}{2} E,}
$$

with $B(z)=\log (z / R)+\phi$. Then, Eq. (22) reduces to the form

$$
\partial_{r_{*}}^{2} \psi+\omega^{2} \psi=U \psi
$$

The potential $U(z)$, obtained this way, diverge at $z=0$ so one must impose $\psi(z=0)=0$. At the horizon $U\left(z=z_{h}\right)=0$, so one expects to find infalling $\psi=$ $e^{-i \omega r_{*}}$ and outgoing $\psi=e^{+i \omega r_{*}}$ wave solutions for Eq. (24). Only the first kind of solutions are physically allowed. The Schödinger-like equation can be expanded near the horizon leading to the following expansion the field solution:

$\psi=e^{-i \omega r_{*}(z)}\left[1+a^{(1)}\left(z-z_{h}\right)+a^{(2)}\left(z-z_{h}\right)^{2}+\ldots\right]$.

One can solve recursively for $a^{(n)}$. The first coefficient obtained is

$a^{(1)}=\frac{\left(2-q^{2} z_{h}^{6}\right)\left(z_{h}\left(\frac{k^{2}}{2-q^{2} z_{h}^{6}}+2 \kappa^{2}\right)-\frac{\operatorname{sech}^{2}\left(\frac{\kappa}{\sqrt{\sigma}}-\frac{1}{M z_{h}}\right)}{M z_{h}^{2}}+\frac{1}{z_{h}}+M\right)}{2\left(q^{2} z_{h}^{6}+i \omega z_{h}-2\right)}$.

This expansion leads to the following form for the infalling boundary conditions for the field and its derivative at the horizon:

$$
\begin{aligned}
E\left(z_{h}\right) & =e^{-i \omega r_{*}\left(z_{h}\right)+\frac{B\left(z_{h}\right)}{2}}, \\
E^{\prime}\left(z_{h}\right) & =\left(-i \omega r_{*}^{\prime}\left(z_{h}\right)+\frac{B^{\prime}\left(z_{h}\right)}{2}+a_{j}^{(1)}\right) E\left(z_{h}\right) .
\end{aligned}
$$

Then one solves Eq. (22) numerically integrating from the horizon, using a method that consists of imposing these infalling boundary conditions and search for complex frequencies that provide solutions vanishing on the boundary $E(z=0)=0$. The results are the quasinormal frequencies and the corresponding solutions are the quasinormal modes, that represent the heavy meson quasistates in the thermal medium.

\section{Entropy}

The solutions for the gravity fields that holographically describe the heavy vector mesons are complex. So, the actual form of the Lagrangian density is $F_{m n}^{*} F^{m n}$. The configuration entropy is calculated from the solutions $v(p, z)$ corresponding to the quasinormal modes $v_{n}(z)$, described in the previous section. One considers the Fourier transform of the energy density $\rho(z)$ in coordinate $z: \tilde{\rho}(k)$. It is convenient, for the computation of the $\mathrm{CE}$, to split $\tilde{\rho}(k)=(C(k)-i S(k)) / \sqrt{2 \pi}$, where

$$
\begin{aligned}
C(k) & =\int_{0}^{z_{h}} \rho(z) \cos (k z) d z, \\
S(k) & =\int_{0}^{z_{h}} \rho(z) \sin (k z) d z .
\end{aligned}
$$

In terms of these components, the modal fraction reads

$$
\widetilde{D \epsilon}(k)=\frac{S^{2}(k)+C^{2}(k)}{\left[S^{2}(k)+C^{2}(k)\right]_{\max }} .
$$

For this one-dimensional case, the CE (5) reads

$$
\tilde{S}=-\int_{-\infty}^{\infty} \widetilde{D \epsilon}(k) \log [\widetilde{D \epsilon}(k)] d k .
$$

\section{RESULTS}

We calculated the DCE for bottomonium and charmonium $1 \mathrm{~S}$ states, considering four representative temperatures. The dissociation occurs in different temperatures for these two heavy mesons. Charmonium $1 \mathrm{~S}$ state, the $\mathrm{J} / \psi$, dissociates at lower values of $\mathrm{T}$, so we chose temperatures ranging from 200 to $500 \mathrm{MeV}$, while for bottomonium 1S state, the $\Upsilon$, that dissociates at higher temperatures, we chose a range from 300 to $600 \mathrm{MeV}$. In Fig. 1, we show plots for the DCE for the bottomonium 1S state. Then, in Fig. 2, we present similar plots for the charmonium $1 \mathrm{~S}$ state. In both cases, one clearly sees that the DCE increases with the temperature and the density. The variation with the 


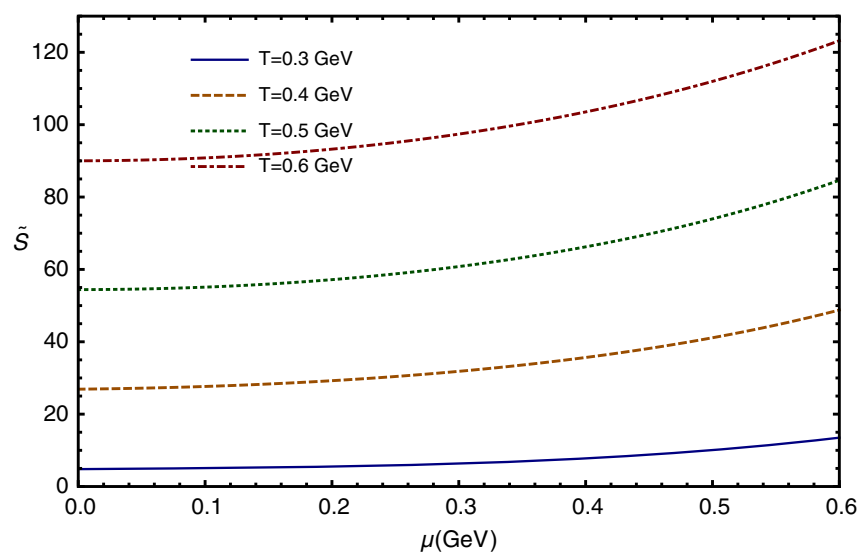

FIG. 1. Differential configuration entropy $\tilde{S}$ as a function of the chemical potential for bottomonium $\Upsilon$ at temperatures: $T=300$ (blue solid line), 400 (brown slashed line), 500 (green dotted line), and $600 \mathrm{MeV}$ (red dash-dotted line).

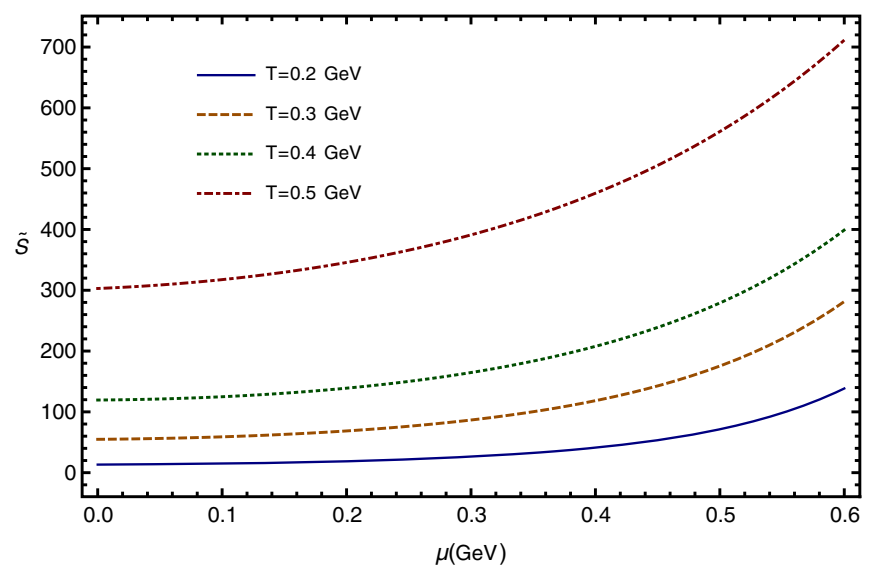

FIG. 2. Differential configuration entropy $\tilde{S}$ as a function of the chemical potential for charmonium $\tilde{S}$ at temperatures: $T=200$ (blue solid line), 300 (brown slashed line), 400 (green dotted line), and $500 \mathrm{MeV}$ (red dash-dotted line).

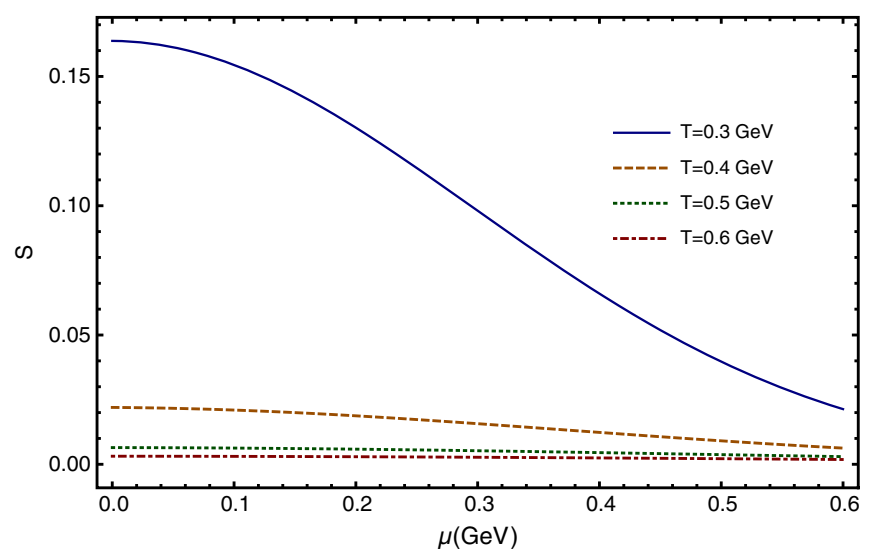

FIG. 3. Dual entropy $S$ as a function of the chemical potential for bottomonium $\Upsilon$ at temperatures: $T=300$ (blue solid line), 400 (brown slashed line), 500 (green dotted line), and $600 \mathrm{MeV}$ (red dash-dotted line).

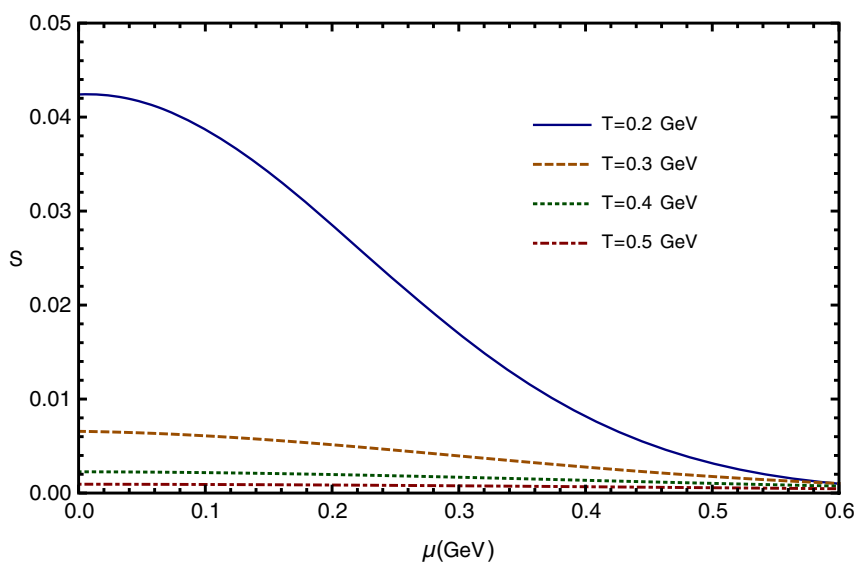

FIG. 4. Dual entropy $S$ as a function of the chemical potential for charmonium $\tilde{S}$ at temperatures: $T=200$ (blue solid line), 300 (brown slashed line), 400 (green dotted line), and $500 \mathrm{MeV}$ (red dash-dotted line).

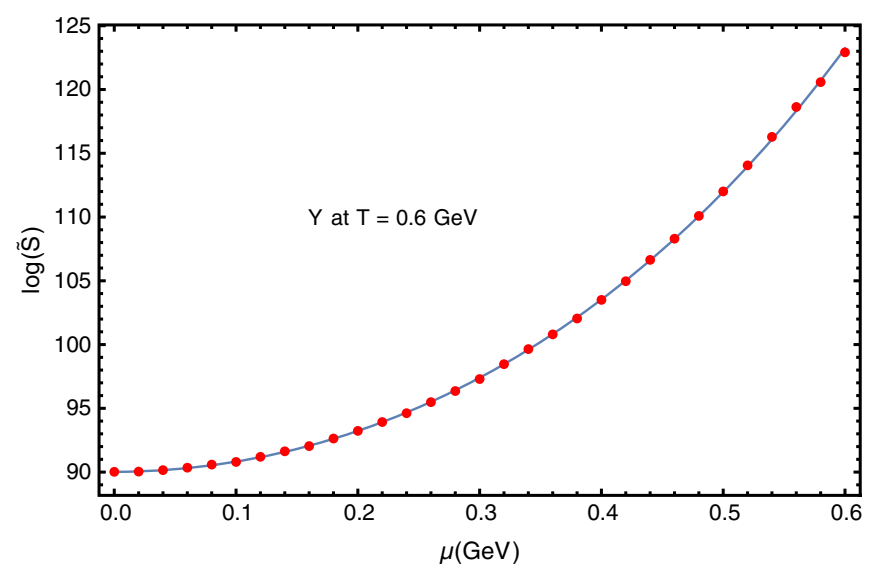

FIG. 5. Logarithm of the differential configuration entropy as a function of $\mu$ for $\Upsilon$ at temperature $600 \mathrm{MeV}$. The dots are the values for $\log (\tilde{S})$ obtained from the model, and the continuous line is the second order polynomial adjust.

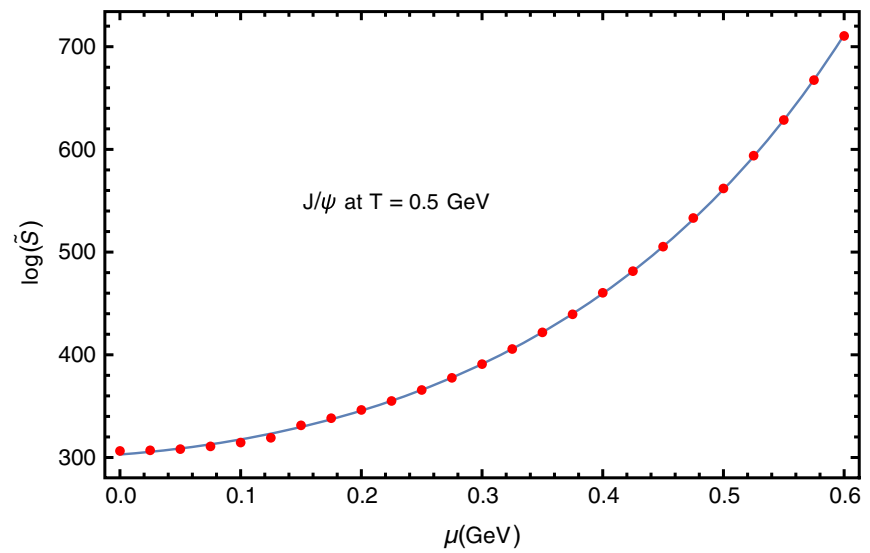

FIG. 6. Logarithm of the differential configuration entropy as a function of $\mu$ for $\mathrm{J} / \psi$ at temperature $500 \mathrm{MeV}$. The dots are the values for $\log (\tilde{S})$ obtained from the model, and the continuous line is the second order polynomial adjust. 
TABLE I. Coefficients $c_{0}, c_{1}$, and $c_{2}$ of Eq. (33) for $\mathrm{J} / \psi$ meson at different temperatures.

Scaling coefficients for $\mathrm{J} / \psi$ differential configurational entropy adjust

\begin{tabular}{lcccc}
\hline $\mathrm{T}(\mathrm{GeV})$ & $c_{0}$ & $c_{1}(\mathrm{GeV})^{-1}$ & $c_{2}(\mathrm{GeV})^{-2}$ & $R_{\mathrm{Adj}}^{2}$ \\
\hline 0.2 & $2.586 \pm 0.022$ & $0.66 \pm 0.11$ & $5.41 \pm 0.13$ & 0.999923 \\
0.3 & $4.0044 \pm 0.0042$ & $0.319 \pm 0.024$ & $4.011 \pm 0.033$ & 0.999986 \\
0.4 & $4.7831 \pm 0.0015$ & $0.1284 \pm 0.0097$ & $3.136 \pm 0.014$ & 0.999996 \\
0.5 & $5.7135 \pm 0.0026$ & $0.279 \pm 0.017$ & $1.906 \pm 0.025$ & 0.9999988 \\
\hline \hline
\end{tabular}

TABLE II. Coefficients $c_{0}, c_{1}$, and $c_{2}$ of Eq. (33) for $\Upsilon$ meson at different temperatures.

Scaling coefficients for $\Upsilon$ differential configurational entropy adjust

\begin{tabular}{lcccc}
\hline $\mathrm{T}(\mathrm{GeV})$ & $c_{0}$ & $c_{1}(\mathrm{GeV})^{-1}$ & $c_{2}(\mathrm{GeV})^{-2}$ & $R_{\text {Adj }}^{2}$ \\
\hline 0.3 & $1.5697 \pm 0.0015$ & $0.184 \pm 0.0010$ & $2.562 \pm 0.015$ & 0.999995 \\
0.4 & $3.2928 \pm 0.0030$ & $0.130 \pm 0.021$ & $1.437 \pm 0.031$ & 0.999975 \\
0.5 & $3.9967 \pm 0.0022$ & $0.002 \pm 0.015$ & $0.006 \pm 0.024$ & 0.999986 \\
0.6 & $4.50002 \pm 0.00055$ & $0.00018 \pm 0.0040$ & $0.8691 \pm 0.0062$ & 0.999999 \\
\hline \hline
\end{tabular}

temperature was analyzed before in Ref. [8], where the same behavior was found. It is known that the dissociation effect of vector mesons inside the plasma is enhanced by the temperature and the density. As the density and (or) the temperature of the plasma increase, the dissociation degree of heavy mesons increases. So, they become more unstable in the sense of their tendency to "melt" in the plasma. So, the results shown in Figs. 1 and 2 for the DCE are consistent with the interpretation that instability corresponds to an increase in the value of this quantity.

We also calculated the dual quantity $S(\mu)$ from Eq. (11), that is the position space conjugate of the DCE; we found a result that is similar to the one obtained in [45]. That means $S(\mu)$ has the opposite behavior, decreasing with $\mu$. We show in Figs. 3 and 4 the value of $S(\mu)$ for bottomonium and charmonium, respectively, for the same temperature ranges.

It is interesting to investigate if the dependence of the DCE on the density $\mu$ can be expressed in the form of a scaling law. As an illustration, we plot in Figs. 5 and 6 the logarithm of the DCE as a function of the density for $\Upsilon$ at $T=600 \mathrm{MeV}$ and for $\mathrm{J} / \psi$ at $T=500 \mathrm{MeV}$, respectively. From the analysis of this kind of plot, for different temperatures, one finds that there is an approximate scaling law of the form

$$
\log (\tilde{S})=c_{0}+c_{1} \mu+c_{2} \mu^{2}
$$

where the coefficients $c_{0}, c_{1}, c_{2}$ depend on the temperature. We show in Tables I and II the values obtained for these parameters at different temperatures and $R_{\text {Adj }}^{2}$ for the polynomial approximation for $\mathrm{J} / \psi$ and $\Upsilon$, respectively.

\section{CONCLUSIONS}

We studied in this paper the variation of the DCE for the $1 \mathrm{~S}$ states of charmonium (the $J / \psi$ ) and bottomonium (the $\Upsilon$ ) as a function of the quark density of the medium, for different temperatures. The results obtained show that the entropy increases with the temperature and the density. This behavior is consistent with the expectation that an increase in the instability of a physical system should correspond to an increase in the DCE. The higher are the values of temperature and (or) density, the higher is the probability that the heavy vector meson will dissociate in the thermal medium. So, increasing the temperature or the density, the quasistates become more unstable against melting in the plasma. This analysis provides one more example of how the DCE characterizes the stability of physical systems.

\section{ACKNOWLEDGMENTS}

The authors are supported by $\mathrm{CNPq}$ - Conselho Nacional de Desenvolvimento Cientifico e Tecnologico (N. R. F. B. by Grant No. 307641/2015-5 and R. d. M. by a graduate fellowship). This work received also support from Coordenacao de Aperfeicoamento de Pessoal de Nivel Superior-Brasil (CAPES)—Finance Code 001. 
[1] M. Gleiser and N. Stamatopoulos, Phys. Lett. B 713, 304 (2012).

[2] M. Gleiser and N. Stamatopoulos, Phys. Rev. D 86, 045004 (2012).

[3] M. Gleiser and D. Sowinski, Phys. Lett. B 727, 272 (2013).

[4] M. Gleiser and N. Jiang, Phys. Rev. D 92, 044046 (2015).

[5] A. E. Bernardini and R. da Rocha, Phys. Lett. B 762, 107 (2016).

[6] A. E. Bernardini, N. R. F. Braga, and R. da Rocha, Phys. Lett. B 765, 81 (2017),

[7] N. R. F. Braga and R. da Rocha, Phys. Lett. B 776, 78 (2018).

[8] N. R. F. Braga, L. F. Ferreira, and R. Da Rocha, Phys. Lett. B 787, 16 (2018).

[9] A. E. Bernardini and R. da Rocha, Phys. Rev. D 98, 126011 (2018).

[10] L. F. Ferreira and R. Da Rocha, Phys. Rev. D 99, 086001 (2019).

[11] A. Alves, A. G. Dias, and R. da Silva, Physica (Utrecht) 420, 1 (2015).

[12] R. A. C. Correa and R. da Rocha, Eur. Phys. J. C 75, 522 (2015).

[13] D. Sowinski and M. Gleiser, J. Stat. Phys. 167, 1221 (2017).

[14] R. Casadio and R. da Rocha, Phys. Lett. B 763, 434 (2016).

[15] R. A. C. Correa, D. M. Dantas, C. A. S. Almeida, and R. da Rocha, Phys. Lett. B 755, 358 (2016).

[16] N. R. F. Braga and R. da Rocha, Phys. Lett. B 767, 386 (2017).

[17] G. Karapetyan, Europhys. Lett. 117, 18001 (2017).

[18] G. Karapetyan, Europhys. Lett. 118, 38001 (2017).

[19] A. Alves, A. G. Dias, and R. da Silva, Braz. J. Phys. 47, 426 (2017).

[20] G. Karapetyan, Phys. Lett. B 781, 201 (2018).

[21] G. Karapetyan, Phys. Lett. B 786, 418 (2018).

[22] M. Gleiser, M. Stephens, and D. Sowinski, Phys. Rev. D 97 , 096007 (2018).

[23] P. Colangelo and F. Loparco, Phys. Lett. B 788, 500 (2019).

[24] C. O. Lee, Phys. Lett. B 790, 197 (2019).

[25] D. Bazeia, D. C. Moreira, and E. I. B. Rodrigues, J. Magn. Magn. Mater. 475, 734 (2019).

[26] C. W. Ma and Y. G. Ma, Prog. Part. Nucl. Phys. 99, 120 (2018).

[27] Q. Zhao, B.Z. Mi, and Y. Li, Int. J. Mod. Phys. B 33, 1950119 (2019).

[28] L. F. Ferreira and R. da Rocha, Eur. Phys. J. C 80, 375 (2020).

[29] A. E. Bernardini and R. da Rocha, Phys. Lett. B 796, 107 (2019).

[30] G. Karapetyan, Europhys. Lett. 129, 18002 (2020).

[31] S. A. Bass, M. Gyulassy, H. Stoecker, and W. Greiner, J. Phys. G 25, R1 (1999).

[32] S. Scherer et al., Prog. Part. Nucl. Phys. 42, 279 (1999).

[33] E. Shuryak, Prog. Part. Nucl. Phys. 62, 48 (2009).

[34] J. Casalderrey-Solana, H. Liu, D. Mateos, K. Rajagopal, and U. A. Wiedemann, Gauge/String Duality, Hot QCD and Heavy Ion Collisions (Cambridge University Press, Cambridge, United Kingdom, 2014).

[35] N. R. F. Braga, M. A. Martin Contreras, and S. Diles, Phys. Lett. B 763, 203 (2016).

[36] N. R. F. Braga, M. A. Martin Contreras, and S. Diles, Eur. Phys. J. C 76, 598 (2016).

[37] N. R. F. Braga and L. F. Ferreira, Phys. Lett. B 773, 313 (2017).

[38] N. R. F. Braga, L. F. Ferreira, and A. Vega, Phys. Lett. B 774, 476 (2017).

[39] N. R. F. Braga and L. F. Ferreira, Phys. Lett. B 783, 186 (2018).

[40] N. R. F. Braga and L. F. Ferreira, J. High Energy Phys. 01 (2019) 082.

[41] N. R. F. Braga and L. F. Ferreira, Phys. Lett. B 795, 462 (2019).

[42] N. R. F. Braga and R. Da Mata, Phys. Lett. B 804, 135381 (2020).

[43] C. E. Shannon, Bell Syst. Tech. J. 27, 379 (1948).

[44] I. Biaynicki-Birula and J. Mycielski, Commun. Math. Phys. 44, 129 (1975).

[45] N. R. F. Braga, Phys. Lett. B 797, 134919 (2019).

[46] P. Colangelo, F. Giannuzzi, and S. Nicotri, Phys. Rev. D 83, 035015 (2011).

[47] P. Colangelo, F. Giannuzzi, S. Nicotri, and V. Tangorra, Eur. Phys. J. C 72, 2096 (2012).

[48] P. Colangelo, F. Giannuzzi, and S. Nicotri, J. High Energy Phys. 05 (2012) 076.

[49] T. Branz, T. Gutsche, V. E. Lyubovitskij, I. Schmidt, and A. Vega, Phys. Rev. D 82, 074022 (2010).

[50] T. Gutsche, V. E. Lyubovitskij, I. Schmidt, and A. Vega, Phys. Rev. D 87, 056001 (2013).

[51] K. B. Fadafan, Eur. Phys. J. C 71, 1799 (2011).

[52] K. B. Fadafan and E. Azimfard, Nucl. Phys. B863, 347 (2012).

[53] K. B. Fadafan and S. K. Tabatabaei, Eur. Phys. J. C 74, 2842 (2014).

[54] S. S. Afonin and I. V. Pusenkov, Phys. Lett. B 726, 283 (2013).

[55] L. A. H. Mamani, A. S. Miranda, H. Boschi-Filho, and N. R. F. Braga, J. High Energy Phys. 03 (2014) 058.

[56] K. Hashimoto, N. Ogawa, and Y. Yamaguchi, J. High Energy Phys. 06 (2015) 040.

[57] Y. Liu and I. Zahed, Phys. Rev. D 95, 056022 (2017).

[58] Y. Liu and I. Zahed, Phys. Lett. B 769, 314 (2017).

[59] A. Ballon-Bayona, G. Krein, and C. Miller, Phys. Rev. D 96, 014017 (2017).

[60] D. Dudal and T. G. Mertens, Phys. Rev. D 97, 054035 (2018).

[61] T. Gutsche, V. E. Lyubovitskij, I. Schmidt, and A. Y. Trifonov, Phys. Rev. D 99, 054030 (2019).

[62] H. Bohra, D. Dudal, A. Hajilou, and S. Mahapatra, Phys. Lett. B 801, 135184 (2020).

[63] Z. q. Zhang and X. Zhu, Phys. Lett. B 793, 200 (2019).

[64] M. A. Martin Contreras and A. Vega, Phys. Rev. D 101, 046009 (2020). 